\title{
Changes in retina's vascular and nervous structures in patients with hypertension
}

\author{
Run-ze Zhao ( $\nabla 13173187 @ q q . c o m$ ) \\ Hermanos Ameijeiras Hospital \\ Melba Márquez Fernández \\ Hermanos Ameijeiras Hospital \\ Maria Cáceres Toledo \\ Hermanos Ameijeiras Hospital \\ Teddy Osmin Tamargo Barbeito \\ Hermanos Ameijeiras Hospital \\ Guo-xun Zhang \\ University of Seville \\ Wen-ting Zhang \\ University of Seville
}

\section{Research Article}

Keywords: Hypertension, ambulatory blood pressure monitoring, hypertensive retinopathy, retinal nerve fiber layer, ganglion cell complex

Posted Date: February 24th, 2021

DOI: https://doi.org/10.21203/rs.3.rs-232650/v1

License: (c) (i) This work is licensed under a Creative Commons Attribution 4.0 International License.

Read Full License 
Changes in retina's vascular and nervous structures in patients with hypertension

Run-ze Zhao ${ }^{a}$, Melba Márquez Fernándeza ${ }^{a}$ Maria Cáceres Toledoa, Teddy Osmin Tamargo Barbeito ${ }^{\mathrm{b}}$, Guo-xun Zhang ${ }^{\mathrm{c}}$, Wen-ting Zhang . $^{\mathrm{c}}$

\section{Abstract}

Introduction: This study aims to analyze the correlation between hypertension (HTN) and changes in the retina's vascular and nervous structures in patients with hypertension.

Method: This was a cross-sectional study, which included a group of 45 subjects who did not suffer from hypertension (HTN) (Group 1), other two groups of 51 patients with controlled (Group 2) and not controlled (Group 3) HTN. Changes in vascular and nervous structures were identified and evaluated by ophthalmoscopy and OCT, respectively. Parametric and nonparametric tests, Post-hoc, Pearson, and Spearman correlation were used, with $p<0.05$ considered statistically significant.

Results: We observed hypertensive retinopathy (HTNR G I and II) in both groups with HTN, with a significant difference $(p=0.023)$. We found a lower average retinal nerve fiber layer (RNFL), the average and minimum ganglion cell complex (GCC) in the patients of groups 2 and 3 than those of group 1. We found a negative and slight correlation between systolic blood pressure (SBP), diastolic blood pressure (DBP), and HTNR with superior GCC thickness in hypertensive patients. 
Conclusions: hypertension correlated to changes in the retina's vascular and nervous structures, and the nervous structure alterations were not visible by ophthalmoscopy but detected by OCT.

Keywords: Hypertension, ambulatory blood pressure monitoring, hypertensive retinopathy, retinal nerve fiber layer, ganglion cell complex

\section{Introduction}

Hypertension (HTN) is one of the significant health problems in contemporary medicine. ${ }^{(1)}$ Globally, the number of hypertensive cases in 2015 has reached 1.13 billion. (2) In the United States, approximately one-third of adults were diagnosed with high blood pressure in 2017. (3) In addition to its high frequency and geographic spread, the importance of hypertension is that elevated blood pressure causes or accelerates changes in the vessel wall that pose a risk for severe vascular disease in the kidney, heart, brain, and eyes. $(2,4,5)$

In the eye, the vessels irrigate the retina, choroids, and the optic nerve are affected. Known changes occur, such as hypertensive retinopathy, hypertensive choroidopathy, and hypertensive optic neuropathy. Choroidopathy and neuropathy are common in hypertensive emergency and malignant hypertension. ${ }^{(5,6)}$. Hypertensive retinopathy is the most studied disorder; several classifications have described, the most widespread being KeithWagener and Barker ${ }^{(7-9)}$

Through ophthalmoscopy in the fundus examination, the vascular and nervous structures of the retina can be visualized. However, it is not the most precise 
method for determining incipient alterations in the retinal nerve fiber layer and depends on the observer's subjective perceptions and variations. Optical coherence tomography (OCT) shows live images of histological changes in the RNFL and the GCC thickness. ${ }^{(10,11)}$

Different publications related to the presence of retinopathy shown as a predictor of cardiovascular disease; ${ }^{(7)}$ the degree of retinopathy and pressure values; ${ }^{13}$ the stage of hypertension and retinal vascular damage; ${ }^{(12)}$ Lee et al. (13) demonstrate the decrease in RNFL thickness in hypertensive patients compared to a non-hypertensive group. However, Akay et al. ${ }^{(14)}$ only observe the GCC thickness decrease, with no variation in RNFL.

This study analyzes blood pressure values and their relationship with changes in the retina's vascular and nervous structures in patients with mild essential systemic hypertension concerning a group without the disease.

\section{Method}

We conducted a cross-sectional study. A total of 147 subjects were included, conformed by three groups, one of 45 who did not suffer from HTN, the other two of 51 with controlled and uncontrolled HTN, respectively. The hypertensive patients were from the specialized hypertension clinic. The non-hypertensive subjects were healthy volunteers, such as some hospital workers and the patients' accompanying. We examined all in the Neuro-ophthalmology clinic of the Hermanos Ameijeiras Hospital. Havana. 
We carried out data collection from March 2018 to September 2019. All the studies carried out in this research complied with the principles of the Declaration of Helsinki. They were approved by the Scientific Council and the Ethics Committee for Research of the Hermanos Ameijeiras hospital. Before the start of the research, the informed consent was signed by all participants. The criteria for inclusion were: age between 18 and 60 years. The hypertensive patients were included because of the ambulatory monitoring of blood pressure (AMBP) according to the ESC/ESH 2018 Guide (which coincides with the Cuban Guide) on diagnosing and treating arterial hypertension. In the nonhypertensive group, the subjects were selected with a routine ophthalmological examination and blood pressure (BP) in normal ranges by the AMBP.

The exclusion criteria were: patients with occlusive lesions of the carotid arteries, diabetes mellitus, or other systemic diseases affecting the vascular system; alcohol addicts, and smokers. As for eye conditions, cases with glaucoma; previous eye surgery (filtering or cataract surgery); with ametropia of more than 4.0 diopter (D) and better corrected visual acuity below 0.8 were excluded, as well as cases with the opacity of the transparent media of the eye that prevented the visualization of the fundus and OCT studies.

We examined the best-corrected visual acuity measurement with the refraction unit (NIDEK). The ophthalmological examination acted on a fundus examination with the ophthalmoscope (Neitz Psu-1). Two ophthalmologists did posterior 
pole photographs, taking into account the classification of Keith-WagenerBarker ${ }^{17}$. According to the patients' statement, we classified the hypertensive disease duration as less than or equal to ten and more than ten years.

All patients and healthy subjects underwent AMBP examination (Hipermax). The technical explained the importance of maintaining their daily life during the test to them. The equipment uses a program that performs a BP measurement every 20 minutes during the daytime, and every 30 minutes at night, for 24 hours.

The measurement of the intraocular pressure (IOP) was made through a Perkins Mk2 manual flattening tonometer. The average of the three measurements obtained the final IOP value at different times (8:30 am, 11:00 am, 1:00 pm), corrected by pachymetry (Ultrasonic Pachymeter NIDEK UP1000). The BP was obtained by the average value of the three AMBP measurements simultaneously with the IOP measurement. The OPP was calculated by the following formula OPP $=2 / 3 \mathrm{MBP}-\mathrm{IOP}(15)$.

The same HTN specialist evaluated all participants. The AMBP values were used to define the three groups mentioned above. Group 1 was made up of healthy subjects. The patients with antihypertensive treatment and controlled hypertension made up group 2. Group 3 was made up of uncontrolled hypertension, despite medical treatment. AMBP values are usually lower than BP measured in the clinic; the diagnostic threshold for hypertension is $\geq 130 / 80$ $\mathrm{mmHg}$ for $24 \mathrm{~h}$. The average daytime BP value is $\geq 135 / 85 \mathrm{mmHg}$ and $\geq 120 / 70$ 
for nighttime BP. (2)

In all cases, we performed OCT on the retinal nerve fiber layer and ganglion cells layer in the macular region after pupil dilation with tropicamide, thus achieving mydriasis that facilitated the visualization of the ocular structures. Cirrus 5000 HD- OCT was used (Carl Zeiss Meditec Inc. Dublin, California, software version: 9.5.0.8712). The RNFL thickness was analyzed with the Optic Disc Cube 200x200 OCT Cirrus scanning protocol, the Macular Tomography Cube 512x128 acquisition protocol was selected to obtain the GCC thickness.

\section{Information processing}

Only one eye was selected in each patient, randomly between the right eye and the left eye and the right one was chosen. The quantitative variables were summarized with the mean and its standard deviation and the $95 \%$ confidence interval. The qualitative variables were presented with absolute numbers and percentages. In the quantitative variables, the Kolmogorov-Smirnov test was applied to look for the existence of normality. Since these variables did not meet the assumption of normality, the Kruskal-Wallis test was applied. When the value of $p$ was a significant difference between the three groups, Mann-Whitney was used to the post-hoc test to look for differences in means between the groups. The ANOVA test was performed for variables with normal distribution, and Scheffer or Games-Howell used the post-hoc test to look for differences between which groups. For qualitative variables, Pearson's Chi-square was applied. Pearson's correlation test was used for two variables with normal 
distribution, and Spearman's correlation was applied when one of the variables did not have a normal distribution.

\section{RESULTS}

we found no significant differences between the groups according to age, sex, and skin color $(p>0.05)$. (Table 1)

Regarding SBP, DBP, and MBP values, we found significant differences $(p<0.001)$ among the three groups. In group 3, We detected higher BP values compared to groups 1 and 2, and we classified them as uncontrolled hypertensive cases.

The IOP values shown within the normal ranges; however, they were higher in group 3, with a significant difference between the three groups $(p<0.001)$, according to the post-hoc statistical analysis, the difference was between group 3 and groups 1 and $2(p=0.020, p=0.017$ respectively). Therefore, we inferred a correlation between a high BP and IOP.

We found a significant $(p<0.001)$ increase in the mean OPP in group $3(56.4 \pm$ 7.5) when comparing the value obtained with the value of group $1(48.8 \pm 4.6)$, considered as standard in this study.

The HTNR GI was the most frequently found in both hypertensive groups, with differences in the vascular structure affectation (HTNR GI and GII) between groups 2 and $3(p=0.023)$. We observed more cases with the HTNR GII in group 3. No significant differences $(p=0.525)$ in hypertensive duration between the 
two hypertensive groups.

Table 1. Demographic and clinical characteristics of the subjects and patients studied

\begin{tabular}{|c|c|c|c|c|c|c|c|}
\hline & $\begin{array}{l}\text { No HTN } \\
(n=45)\end{array}$ & $\begin{array}{c}\text { Controlled } \\
\qquad \text { HTN } \\
(n=51)\end{array}$ & $\begin{array}{l}\text { No controlled } \\
\text { HTN } \\
\quad(n=51)\end{array}$ & p & $\mathbf{p}^{\star \star \star}$ & $\begin{array}{l}P^{\star *} \\
* *\end{array}$ & $\mathbf{P}^{\star \star \star \star \star \star}$ \\
\hline Age,y & $43,9 \pm 10,7$ & $47,5 \pm 7,5$ & $46,9 \pm 8,9$ & $0,390^{*}$ & & & \\
\hline$M / F, n$ & $28 / 17$ & $21 / 30$ & $27 / 24$ & $0,117^{\star *}$ & & & \\
\hline $\begin{array}{l}\text { Skin } \\
\text { color }\end{array}$ & 25/2/ 18 & $21 / 10 / 20$ & $22 / 14 / 15$ & $0,050^{\star *}$ & & & \\
\hline W/B/M & & & & & & & \\
\hline $\begin{array}{c}\text { SBP } \\
\mathrm{mmHg}\end{array}$ & $126,0 \pm 11,9$ & $132,5 \pm 11,5$ & $145,8 \pm 16,1$ & $<0,001^{\star}$ & $<0,001$ & 0.003 & $<0,001$ \\
\hline $\begin{array}{c}\text { DBP } \\
\mathrm{mmHg}\end{array}$ & $81,1 \pm 7,7$ & $84,7 \pm 9,6$ & $90,5 \pm 10,5$ & $<0,001^{*}$ & $<0,001$ & 0,062 & 0,004 \\
\hline $\begin{array}{c}\text { MBP } \\
\mathrm{mmHg}\end{array}$ & $96,0 \pm 8,0$ & $100,7 \pm 8,9$ & $109,0 \pm 10,9$ & $<0,001^{\star}$ & $<0,001$ & 0,007 & $<0,001$ \\
\hline $\begin{array}{c}\text { IOP } \\
\mathrm{mmHg}\end{array}$ & $15,3 \pm 1,3$ & $15,2 \pm 2,0$ & $16,2 \pm 1,8$ & $0,023^{*}$ & 0,020 & 0,867 & 0.017 \\
\hline $\begin{array}{c}\text { OPP } \\
\mathrm{mmHg}\end{array}$ & $48,8 \pm 4,6$ & $51,9 \pm 5,8$ & $56,4 \pm 7,5$ & $<0,001^{\star}$ & $<0,001$ & 0.003 & 0.001 \\
\hline $\begin{array}{c}\text { HR } 0 / \text { I / } \\
\text { II }\end{array}$ & & $18 / 30 / 3$ & $8 / 33 / 10$ & $0,023^{\star *}$ & & & \\
\hline $\begin{array}{c}\leq 10 />10 \\
\text { years }\end{array}$ & & $36 / 15$ & $33 / 18$ & $0,525^{\star \star}$ & & & \\
\hline
\end{tabular}

M/F: male/female, B/W/M: white/black/mestizo, SBP: systolic blood pressure, DBP: diastolic blood pressure, MAP: mean blood pressure, IOP: intraocular pressure, OPP: ocular perfusion pressure, HTNR: hypertensive retinopathy, *: Kruskal-Wallis test, Chi-square test (X2), ${ }^{* * *}$ : post hoc test (Mann-Whitney) between $\mathrm{G} 1$ and $\mathrm{G} 3$, ${ }^{* * * *}$ : post hoc test (Mann-Whitney) between $\mathrm{G} 1$ and $\mathrm{G}$ $2{ }^{* * * *}$ : post hoc test (Mann-Whitney) between G 2 and G 3. 
Table 2 shows the RNFL study by OCT in the three groups. We found a significant difference in the average RNFL values between the three groups $(p=0.023)$, where the RNFL thickness of groups 2 and 3 was demonstrated thinker than that of group 1. The difference between the means was between group 1 and group $2(p=0.040)$.

Table 2. Characteristics of retinal nerve fiber layer (RNFL) in OCT

\begin{tabular}{lrrrrr}
\hline OCT parameter & Group 1 & Group 2 & Group 3 & p & $\mathrm{p}^{* * *}$ \\
& (Mean \pm SD) & (Mean \pm SD) & (Mean \pm SD) & & \\
Average RNFL & $101,5 \pm 7,6$ & $96,5 \pm 10,5$ & $97,0 \pm 10,5$ & $\mathbf{0 , 0 2 3} \mathbf{3}^{*}$ & $\mathbf{0 , 0 4 0}$ \\
Superior RNFL & $125,4 \pm 11,9$ & $123,8 \pm 15,3$ & $118,5 \pm 16,6$ & $0,058^{*}$ & \\
Nasal RNFL & $78,5 \pm 15,0$ & $76,3 \pm 13,3$ & $76,2 \pm 13,3$ & $0,670^{* *}$ & \\
Inferior RNFL & $134,7 \pm 13,7$ & $127,5 \pm 18,9$ & $129,2 \pm 21,3$ & $0,143^{*}$ & \\
Temporal RNFL & $62,6 \pm 9,2$ & $60,8 \pm 9,7$ & $59,5 \pm 8,7$ & $0,258^{* *}$ & \\
\hline
\end{tabular}

RNFL: retinal nerve fiber layer, $\quad$ *: ANOVA, ${ }^{* *}$ : Kruskal Wallis test, ${ }^{* * *}$ : post-hoc test (Scheffé) between group 1 and group 2

Table 3 shows the study of GCC by OCT in the three groups. We found significant differences in both the average $(p=0.032)$ and minimum values $(p=0.019)$, with a significant decrease in the average GCC thickness in groups 2 and 3 to group $1(p=0.031$ and $p=0.046$ respectively). In average minimum GCC value, the significant difference was between group 1 and group 3 $(p=0.024)$ 
Table 3. Characteristics of the ganglion cell complex (GCC) in OCT

\begin{tabular}{lllllll}
\hline GCC Parameter & group 1 & group 2 & group 3 & $p$ & $P^{\star \star \star}$ & $P^{* \star \star \star}$ \\
& (Mean \pm & (Mean \pm & (Mean \pm & & & \\
& SD) & SD) & SD) & & & \\
\hline Average & $85,7 \pm 4,6$ & $83,0 \pm 5,7$ & $82,8 \pm 7,3$ & $\mathbf{0 , 0 3 2 *}$ & $\mathbf{0 , 0 3 1}$ & $\mathbf{0 , 0 4 6}$ \\
Minimum & $82,1 \pm 5,9$ & $79,2 \pm 6,2$ & $77,7 \pm 9,9$ & $\mathbf{0 , 0 1 9 *}$ & $\mathbf{0 , 0 2 4}$ \\
Superior & $85,8 \pm 5,8$ & $85,1 \pm 7,1$ & $83,2 \pm 8,9$ & $0,204^{*}$ & & \\
Superotemporal & $83,1 \pm 6,5$ & $82,0 \pm 6,4$ & $81,7 \pm 8,2$ & $0,624^{*}$ & & \\
Inferotemporal & $83,6 \pm 5,8$ & $82,2 \pm 5,9$ & $81,0 \pm 9,6$ & $0,236^{*}$ & & \\
Inferior & $82,8 \pm 5,9$ & $80,6 \pm 6,6$ & $80,3 \pm 8,6$ & $0,203^{*}$ & & \\
Inferonasal & $84,9 \pm 5,6$ & $83,9 \pm 7,0$ & $82,9 \pm 7,7$ & $0,344^{*}$ & & \\
Superonasal & $86,9 \pm 5,0$ & $86,1 \pm 7,3$ & $84,5 \pm 6,9$ & $0,198^{* *}$ & & \\
\hline
\end{tabular}

*: ANOVA, ${ }^{* *}$ : Kruskal Wallis test, ${ }^{* * *}$ : post-hoc test (Games-Howell) between group 1 and group 2, ${ }^{* * *}$ : post-hoc test (Games-Howell) between group 1 and group 3

The correlation test between the following variables was obtained: SBP, DBP, and IOP resulting from the retina's vascular and nervous structures and the time of evolution in hypertensive cases (See table 4). We found no correlation between IOP and the rest of the variables, which may attribute it to the fact that the IOP values remained within the normal range.

The correlations between SBP, DBP, and HTNR were significant positively $(p<0.001)$. It illustrated a stronger correlation between SBP and HTNR than between DBP and HTNR. SBP, DBP, and HTNR were significantly correlated with superior GCC thickness, negatively and slightly. The correlation between DBP and superotemporal GCC thickness was significant $(p=0.035)$, negatively 
and slightly, which demonstrated that the PAD's increase is related to a slight GCC decrease in the referred retinal quadrant. We also found a negative correlation between the thickness of the nasal RNFL and the duration of hypertension $(p=0.018)$, which means that the RNFL decrease of that sector could be observed in patients with more than ten years of evolution of hypertension.

Table 4. Significant correlations between SBP, DBP, HTNR, Duration of HTN, GCC, and RNFL

\begin{tabular}{lllll}
\hline & SBP & DBP & HR & Duration \\
\hline Superior GCC & & & & \\
$\mathbf{R}$ & $-0,217$ & -0.242 & -0.230 & \\
$\mathbf{P}$ & $0,028^{\mathrm{b}}$ & $0.014^{\mathrm{a}}$ & $0.020^{\mathrm{b}}$ & \\
Superotemporal & & & & \\
GCC & & & & \\
$\mathbf{R}$ & & -0.209 & & \\
$\mathbf{P}$ & & $0.035^{\mathrm{a}}$ & \\
Nasal RFNL & & & \\
$\mathbf{R}$ & & & \\
$\mathbf{P}$ & & & 0.233 \\
$\mathbf{H R}$ & & & $0.018^{\mathrm{b}}$ \\
$\mathbf{R}$ & 0.389 & 0.291 & \\
$\mathbf{P}$ & $<0,001^{\mathrm{b}}$ & $<0,001^{\mathrm{b}}$ & \\
\hline
\end{tabular}

a: Pearson correlation, b: Spearman correlation

\section{Discussion}

In the present study, increased blood pressure correlated with damage to the retina's vascular and nervous structures in hypertensive patients compared to non-hypertensive people.

The patients presented hypertension classified as mild and moderate; most of 
them had less than ten years of evolution, therefore lower damage of the vascular endothelium, which could explain the increase of the PPO values in the hypertensive cases concerning the healthy people. Kanadani et al.(16) reported elevated PPO values in hypertensive patients compared to the healthy population. The vessels' self-regulating mechanism tries to maintain a constant ocular blood flow despite the change in PPO. (15) In patients with chronic hypertension, increased peripheral resistance and small-vessel disease may also reduce optic nerve head perfusion. $(15,17)$

The Los Angeles Latino Eye Study ${ }^{(18)}$ shows that a ten $\mathrm{mmHg}$ increase in the SBP and DBP is equivalent to an elevation of the IOP by 0.33 and $0.44 \mathrm{~mm} \mathrm{Hg}$, respectively. This result coincides with our result.

In the present study, the HTNR G I was the most frequent fundoscopic finding in the hypertensive cases; due to the arguments previously exposed regarding the type of hypertension and its duration, we found more cases with HTNR GII in group 3 concerning group 2. It also illustrated the positive correlation between SBP and DBP alteration with the degree of hypertensive retinopathy.

The alterations in the retinal vessels caused by hypertension have been well studied for several years, demonstrated by the existing publications on hypertensive retinopathy. ${ }^{(12,19)}$ Cáceres et al. ${ }^{(20)}$ propose that the alterations in the arteriovenous crossings and the vessels' tortuosity are more related to the evolution of the HTN than to its severity. Akay ${ }^{(14)}$ and collaborators consider that HTA causes pathological changes in the retinal vessels. In the initial stage, 
there is a generalized narrowing of the retinal arterioles due to the selfregulation mechanisms that cause a vessel spasm, followed by sclerosis and later exudation characterized by micro-aneurysms and hemorrhages.

Analysis of GCC can give more precise information than RNFL and can be an early indicator of damage to the retinal nerve structure, which is crucial to avoid irreversible damage leading to blindness ${ }^{(21)}$. This study detected a decrease in GCC average and minimum values in both hypertensive groups; however, only with less RNFL average value in the hypertensive group controlled concerning the non-hypertensive; the small sample of three groups may be the principal reason for it.

Lee et al ${ }^{(13)}$ show that in patients with systemic hypertension and severe hypertensive retinopathy, a significant decrease in the RNFL thickness and the retina's central macula thickness occurs to a group of healthy subjects.

Akay et al. (14) ratify a correlation between clinical parameters such as blood pressure variations and their duration to reduce the GCC's superior and inferior temporal sectors. Although we do not observe different sectors among the three groups in this study, a negative correlation of SBP, DBP, and HTNR with GCC's superior sector was identified in hypertensive patients.

Based on the above, we inference that vascular alterations, arteriosclerosis, and blood flow disturbances caused by HTN could lead to a deficiency in retinal perfusion with the possible evolution of both structures' ischemia.

It is one of the leading causes of non-arteritic ischemic optic neuropathy ${ }^{(22)}$. Our 
research found the correlation between systemic and ocular vascular parameters of patients with HTN and its repercussion on RNFL and GCC, which lays the foundations for future study of the retinal ischemic alterations in these patients.

This research focused on patients with mild and moderate hypertension, besides an average age of 47 years; therefore, both vascular and nervous structure alterations are incipient. The fundamental contribution of this research will give a guide to the ophthalmologists and clinics of the Primary Health Care $(\mathrm{PHC})$ and other levels, as for the control and adequate treatment of these patients, to avoid damages in the retina's vascular and nervous structures, which can be progressive and lead to irreversible blindness if they are not diagnosed and treated in an initial stage.

The present study does not contemplate these patients' evolutionary follow-up, which could be the basis for future prospective research. Also, a larger sample size is necessary.

\section{Conclusions}

We found the repercussion of the alterations of the blood pressure in the affectation of the retina's vascular and nervous structures, which were not yet visible by the ophthalmoscopy detected by OCT. OCT's importance in diagnosing retinal nerve structures in patients with HTN was observed earlier in GCC than in FNRL. 


\section{Availability of data and materials}

All data generated or analyzed to support the findings of this study are available in the paper without restriction. The raw data during this study are available from the corresponding author on reasonable request.

\section{References}

1. Oparil S, Acelajado MC, Bakris GL, Berlowitz DR, Cífková R, Dominiczak AF, et al. Hypertension. Nat Rev Dis Primers. 2018;4:18014.

2. $2018 \mathrm{ESC} / \mathrm{ESH}$ Guidelines for the management of arterial hypertension. Rev Esp Cardiol (Engl Ed). 2019;72(2):160.

3. Fang J, Gillespie C, Ayala C, Loustalot F. Prevalence of Self-Reported Hypertension and Antihypertensive Medication Use Among Adults Aged $\geqslant 18$ Years - United States, 2011-2015. MMWR Morb Mortal Wkly Rep. 2018;67(7):219-24.

4. Bhargava M, Ikram MK, Wong TY. How does hypertension affect your eyes? J Hum Hypertens. 2012;26(2):71-83.

5. Henderson AD, Bruce BB, Newman NJ, Biousse V. Hypertension-related eye abnormalities and the risk of stroke. Rev Neurol Dis. 2011;8(1-2):1-9.

6. Stacey AW, Sozener CB, Besirli CG. Hypertensive emergency presenting as blurry vision in a patient with hypertensive chorioretinopathy. Int $\mathrm{J}$ Emerg Med. 2015;8:13.

7. Harjasouliha A, Raiji V, Garcia Gonzalez JM. Review of hypertensive 
retinopathy. Dis Mon. 2017;63(3):63-9.

8. Rodríguez NA, Zurutuza A. [Ophthalmological manifestations of arterial hypertension]. An Sist Sanit Navar. 2008;31 Suppl 3:13-22.

9. Yıldııım T, Özkan S, Yılmaz Ö, Yavuz B. Increased rate of any retinopathy risk in patients with masked hypertension. Clin Exp Hypertens. 2020;42(6):47982.

10. Miki A, Ikuno Y, Jo Y, Nishida K. Comparison of enhanced depth imaging and high-penetration optical coherence tomography for imaging deep optic nerve head and parapapillary structures. Clin Ophthalmol. 2013;7:1995-2001. 11. Mwanza JC, Warren JL, Budenz DL. Utility of combining spectral domain optical coherence tomography structural parameters for the diagnosis of early glaucoma: a mini-review. Eye Vis (Lond). 2018;5:9.

12. Cheung CY, Ikram MK, Sabanayagam C, Wong TY. Retinal microvasculature as a model to study the manifestations of hypertension. Hypertension. 2012;60(5):1094-103.

13. Lee HM, Lee WH, Kim KN, Jo YJ, Kim JY. Changes in thickness of central macula and retinal nerve fibre layer in severe hypertensive retinopathy: a 1year longitudinal study. Acta Ophthalmol. 2018;96(3):e386-e92.

14. Akay F, Gündoğan FC, Yolcu U, Toyran S, Tunç E, Uzun S. Retinal structural changes in systemic arterial hypertension: an OCT study. Eur $\mathrm{J}$ Ophthalmol. 2016;26(5):436-41.

15. Caprioli J, Coleman AL. Blood pressure, perfusion pressure, and glaucoma. 
Am J Ophthalmol. 2010;149(5):704-12.

16. Kanadani FN, Figueiredo CR, Miranda RM, Cunha PL, TC MK, Dorairaj S. Ocular Perfusion Pressure and Pulsatile Ocular Blood Flow in Normal and Systemic Hypertensive Patients. J Curr Glaucoma Pract. 2015;9(1):16-9.

17. Cantor E, Méndez F, Rivera C, Castillo A, Martínez-Blanco A. Blood pressure, ocular perfusion pressure and open-angle glaucoma in patients with systemic hypertension. Clin Ophthalmol. 2018;12:1511-7.

18. Memarzadeh F, Ying-Lai M, Chung J, Azen SP, Varma R. Blood pressure, perfusion pressure, and open-angle glaucoma: the Los Angeles Latino Eye Study. Invest Ophthalmol Vis Sci. 2010;51(6):2872-7.

19. Duarte T, Gonçalves S, Brito R, Sá C, Marinheiro R, Fonseca M, et al. Relationship between nocturnal blood pressure profiles and the presence and severity of hypertensive retinopathy. Rev Port Cardiol. 2018;37(2):169-73.

20. Cáceres Toledo M, Cáceres Toledo O, Cordies Jackson L. Hipertensión arterial y retinopatía hipertensiva, su comportamiento en un área de salud. Rev Cubana Med 2000;39 (4):210-6.

21. Rebolleda G, Diez-Alvarez L, Casado A, Sánchez-Sánchez C, de Dompablo E, González-López JJ, et al. OCT: New perspectives in neuroophthalmology. Saudi J Ophthalmol. 2015;29(1):9-25.

22. Miller NR, Arnold AC. Current concepts in the diagnosis, pathogenesis, and management of nonarteritic anterior ischaemic optic neuropathy. Eye (Lond). 2015;29(1):65-79. 


\section{Acknowledgments}

This study was supported by the Neuro-ophthalmology and Hypertension

Service, Hermanos Ameijeiras Hospital, Havana, Cuba.

\section{Ethics declarations}

\section{Ethics approval and consent to participate}

This study received ethical approval from the Scientific Council and the Ethics

Committee for Research of the Hermanos Ameijeiras hospital and adhered to the tenets of the Declaration of Helsinki. Before the start of the research, the informed consent was signed by all participants. All the data was recorded and stored in compliance with ethical and data protection guidelines.

\section{Consent for publication}

Not applicable.

\section{Competing interests}

Non-financial competing interests.

\section{Author information}

\section{Affiliations}

a. Department of Ophthalmology, Hermanos Ameijeiras Hospital, Havana,

Cuba. Run-ze Zhao, Melba Márquez Fernández, Maria Cáceres Toledo

b. Department of research, Hermanos Ameijeiras Hospital, Havana, Cuba. Teddy Osmin Tamargo Barbeito

c. Doctoral School, University of Seville, Spain. Guo-xun Zhang, Wen-ting Zhang 
Correspondence: Run-ze Zhao. Department of Ophthalmology, Hermanos Ameijeiras Hospital, Calle San Lázaro \# 701 esq. a Belascoaín, Centro Habana, La Habana, C.P:10400, Cuba. Tel: 0053 54815610. Email : 313173187@qq.com

\section{Authors' contributors}

Zhao Run-ze conceived and designed the study and is responsible for data collection and analysis, and drafted it. Márquez Fernández Melba, Cáceres Toledo Maria reviewed and revised the paper. Zhang Guo-xun, Zhang Wen-ting Tamargo Barbeito Teddy Osmin assists in data analysis and provide advice. All authors read and approved the manuscript.

\section{Funding}

The authors have not declared a specific grant for this research from any funding agency in the public, commercial, or not-for-profit sectors. 